\title{
The Ischial Spine Sign
}

\section{Does Pelvic Tilt and Rotation Matter?}

\author{
Diganta K. Kakaty MD, Andreas F. Fischer MD, \\ Harish S. Hosalkar MD, MBMS (Orth), FCPS (Orth), DNB (Orth), \\ Klaus A. Siebenrock MD, Moritz Tannast MD
}

Received: 29 January 2009/Accepted: 22 July 2009/Published online: 7 August 2009

(C) The Association of Bone and Joint Surgeons (B) 2009

\begin{abstract}
Although the ischial spine sign (ISS) has been advocated to detect acetabular retroversion, it is unknown whether the sign is valid on anteroposterior (AP) pelvic radiographs with tilted or rotated pelves. We therefore evaluated reliability of the ISS as a tool for diagnosing acetabular retroversion in the presence of considerable pelvic tilt and/or malrotation. We obtained radiographs of 20 cadaver pelves in 19 different malorientations resulting in 380 pelvis images (760 hips) for evaluation. In addition, 129 clinical radiographs of patients' hips that had varying pelvis orientations were reviewed. We found an overall sensitivity of $81 \%(90 \%)$, specificity of $70 \%(71 \%)$, positive predictive value of $77 \%(80.7 \%)$, and negative predictive value of $75 \%$ ( $85 \%$ ) in the cadaver (patient) hips. Our data suggest the ISS is a valid tool for diagnosing acetabular retroversion on plain radiographs taken using a standardized technique regardless of the degree of pelvic tilt and rotation.
\end{abstract}

One or more of the authors (KAS, MT) has received funding from the National Center for Competence in Research "Co-Me" of the Swiss National Science Foundation.

Each author certifies that his or her institution has approved the human protocol for this investigation and that all investigations were conducted in conformity with ethical principles of research. This work was performed at the University of Bern, Bern, Switzerland.

D. K. Kakaty, A. F. Fischer, K. A. Siebenrock, M. Tannast ( $\square)$ Department of Orthopaedic Surgery, Inselspital, University of Bern, Murtenstrasse, 3010 Bern, Switzerland e-mail: moritz.tannast@insel.ch

H. S. Hosalkar

Department of Orthopaedic Surgery, Hospital of the University of Pennsylvania, Philadelphia, PA, USA

\section{Introduction}

Retroversion of the acetabulum is a specific variation in morphologic features of the hip in which the cranial portion of the acetabulum is posteriorly oriented [9]. This can lead to dynamic pincer impingement between the anterior femoral head-neck junction and the prominent anterolateral acetabular rim thereby predisposing the hip to early degenerative changes, including labral and cartilage damage and eventual osteoarthritis [3,5]. There are strong indicators that a considerable number of hips with acetabular retroversion are the result of rotation of the entire acetabular complex [6-8].

Acetabular retroversion sometimes can be difficult to identify on AP pelvic radiographs $[1,7]$. The ISS, however, reportedly provides a more easily detectable radiographic indicator of acetabular retroversion [7]. The sign is considered present if the projected triangular shape of the ischial spine protrudes and is visible medially to the pelvic brim. Kalberer et al. pointed out that the ISS had a sensitivity and specificity greater than $90 \%$ in detecting retroversion of the acetabulum [7]. However, in their methodology, they excluded approximately $85 \%$ of their total radiographs because of concerns of inadequate pelvic tilt and/or rotation. Although it is well understood that the degree of apparent acetabular retroversion as observed on a planar projection of the pelvis can vary with large changes in pelvic tilt and rotation [10], the clinical reliability of the ISS in detecting retroversion in conditions of pelvic rotation and/or tilt is unknown.

The aims of our study therefore were twofold: (1) to systematically investigate in a cadaver setup the diagnostic performance, sensitivity, sensibility, and positive and negative predictive values of the ISS as an indicator for apparent acetabular retroversion with change of pelvic 
orientation (tilt and rotation); and (2) to assess the sensitivity, sensibility, and positive and negative predictive values of the ISS as an indicator for apparent acetabular retroversion on patient radiographs having suboptimal orientation of their pelvis.

\section{Material and Methods}

There were two parts to our study. The first was an experimental cadaveric study and the second a retrospective radiographic review of clinical cases. The study was approved by the Institutional Review Board for medical studies. In the initial experimental cadaveric study, we obtained 20 dry cadaver pelves ( 10 male, 10 female). None of the cadaveric specimens had any obvious asymmetries, dysplastic, neoplastic, or posttraumatic changes. There were no exclusion criteria for an apparent acetabular retroversion. The rims of the acetabula on each cadaver specimen were marked with a $1.25-\mathrm{mm}$ metal wire. Thus, there were a total of 40 acetabula for evaluation. In this series, $68 \%$ of all hips were retroverted in the neutral orientation. This is markedly higher than the incidence of $5 \%$ reported in the normal population [5]. We used a custommade holding device with radiolucent brackets to clamp the pelvis between the acetabula thereby allowing tilting around the interacetabular axis and rotation around the longitudinal axis in graded $\left(1^{\circ}\right)$ steps (Fig. 1). Positive tilt was defined with the pelvis being tilted forward. Positive rotation was defined with the pelvis being tilted to the right (Fig. 1).

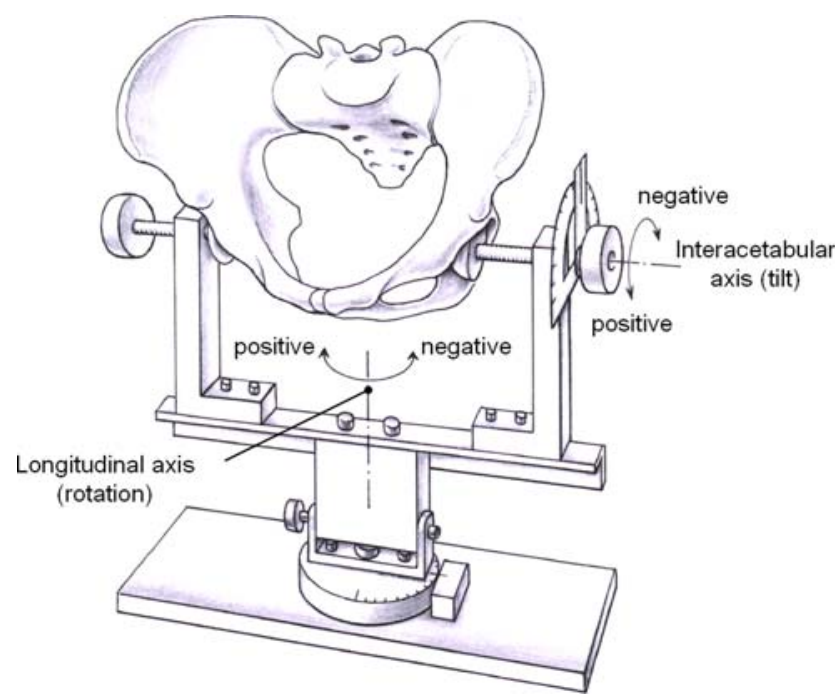

Fig. 1 A diagrammatic illustration shows the holding device used in the cadaver experiments. It allows rotation around the interacetabular (tilt) and longitudinal axis (rotation).
The pelves initially were placed in a neutral orientation. A neutral tilt was defined by a pelvic inclination of $60^{\circ}[2$, 14]. We defined pelvic inclination as the angle between a horizontal line and a line connecting the upper border of the symphysis with the sacral promontory $[2,14]$ as measured on a lateral pelvic radiograph. This lateral pelvic radiograph is taken with the xray beam perpendicular to the patient (similar to a lateral view of the lumbar spine) with the xray beam centered on the greater trochanter in patients or the acetabular center for the cadaver experiments. Rotation around the longitudinal axis was defined as neutral when the projected horizontal distance $b$ between the middle of the sacrococcygeal joint and the symphysis was $0 \mathrm{~mm}$ (Fig. 2) [13]. We then obtained serial digital AP radiographs for different pelvic orientations (Fig. 2); each specimen was tilted sequentially around the two axes (interacetabular and longitudinal; Fig. 1) in graded increments of $3^{\circ}$ at a time. The maximum tilt amplitude of $\pm 12^{\circ}$ and the maximum rotation amplitude of $\pm 9^{\circ}$ were chosen according to the possible range of pelvic malorientation reported in a previous study [10]. This resulted in eight tilted positions and six rotated orientations for each of the 20 pelves. Finally, to test the ISS in cases with combined malpositioning of tilt and rotation, we acquired radiographs of each pelvis with all possible combinations of $\pm 6^{\circ}$ tilt and $\pm 6^{\circ}$ rotation, resulting in four additional radiographs. This resulted in a set of 19 radiographs (eight tilted, six rotated, four combined, and one neutral orientation) for each pelvis. Eventually, there were a total of 380 pelvis radiographs (760 hip projections) for evaluation.

The radiographs were performed in a standardized manner [11, 12]. The film focus distance was $120 \mathrm{~cm}$. The center of the radiographic beam was directed to the midpoint between the symphysis and a line connecting the two anterior-superior iliac spines. The center beam was repositioned before obtaining radiographs of individual acetabulum. We assessed two parameters in all cases, the presence of a crossover sign and the ISS. The crossover sign was considered present when the anterior rim just inferior to the most lateral edge of the acetabulum was projected more lateral than the posterior rim but in the distal part crossed the posterior rim and became more medial to the latter. The ISS was present if the ischial spine shadow extended medially to the pelvic inlet. To detect any interobserver and intraobserver variations for the cadaver study, we selected a random sample of 30 blinded radiographs from the 380 total and they were analyzed twice by two independent observers (DKK, AFF) at an interval greater than 1 week (to minimize recall bias error). For the ISS, we computed an intraobserver kappa value of 0.95 for Observer 1 and 0.93 for Observer 2. The intraobserver kappa value for the crossover sign for both observers was 1.0. Interobserver kappa value for the crossover sign was 1.0 and for the ISS was 0.94. 
Fig. 2A-D (A) An illustration and $(\mathbf{B})$ a corresponding radiograph show a cadaveric pelvis with excessive tilt. (C) This illustration and (D) corresponding radiograph show excessive rotation. Good accordance is present between the ischial spine and the crossover sign. $\mathrm{AW}=$ anterior wall; $\mathrm{PW}=$ posterior wall; IS = ischial spine; arrows = crossover sign. Distance $b$ as the projected horizontal distance between the middle of the symphysis and the sacrococcygeal joint reflects the change of rotation. Optimally without rotation, distance $\mathrm{b}$ is equal to $0 \mathrm{~mm}$.
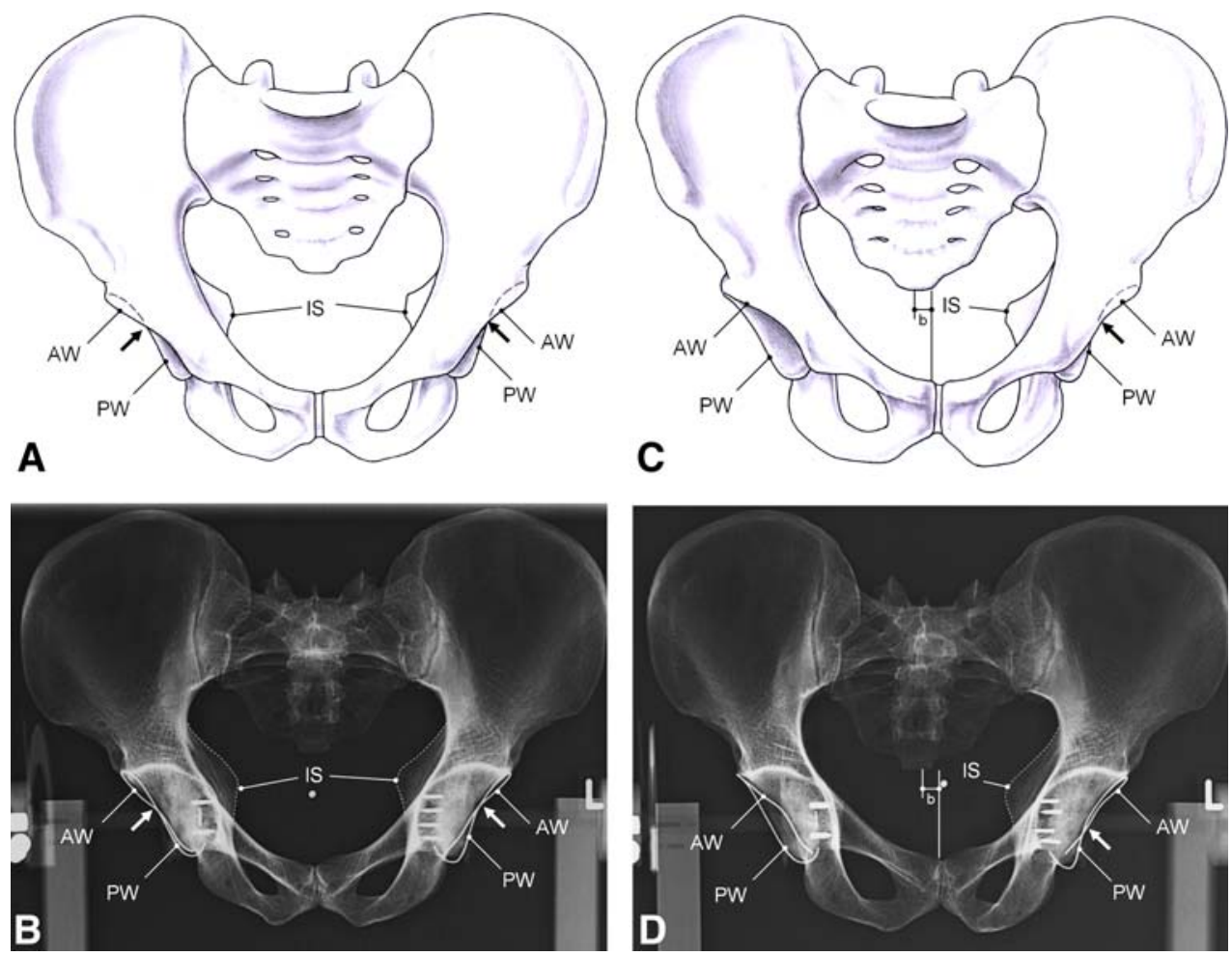

In the retrospective clinical study, we reviewed the digital AP pelvis radiographs of 113 consecutive patients (129 hips, 16 bilateral) who had documented idiopathic femoroacetabular impingement and had presented to our outpatient clinic. Various pelvic orientations were noted in this relatively young patient group (Table 1). The diagnosis of femoroacetabular impingement was based on previously described clinical and radiographic parameters $[3,12]$. The radiographs were taken as per the described standardized

Table 1. Demographic data of the clinical radiographic series

\begin{tabular}{ll}
\hline Parameter & Value \\
\hline Number of patients (hips) & $113(129)$ \\
Number of bilateral hips & 16 \\
Age (years) & $35.1 \pm 11.5(15.3-61.3)$ \\
Gender (percent male of all hips) & 59.7 \\
Side (percent right of all hips) & 60.4 \\
Weight (kg) & $72.9 \pm 16.7(47.3-133)$ \\
Height (m) & $1.70 \pm 0.07(1.4-1.85)$ \\
Body mass index (kg/m ${ }^{2}$ ) & $24.9 \pm 5.0(18.6-41.9)$ \\
Tilt around the interacetabular axis & $5.2 \pm 6.5(-10-19)$ \\
$\quad$ (degrees) & \\
Rotation around the longitudinal axis & $0.3 \pm 2.3(-9.7-5.2)$ \\
$\quad$ (degrees) & \\
$\quad$ Apparent retroversion on radiographs & 87.4 \\
$\quad$ (percent of all hips) & \\
\hline
\end{tabular}

Values of continuous parameters are expressed as mean \pm standard deviation with ranges in parentheses. technique in terms of xray beam centering and film-focus distance $[10,11]$. The patients were placed in a supine position with their legs internally rotated approximately $15^{\circ}$ to $20^{\circ}$ thereby compensating for femoral anteversion. In this study, we did not exclude any patients based on malrotation of the pelvis. The exact tilt and rotation of the patient pelvis were determined based on the vertical and horizontal distance of the sacrococcygeal joint and the symphysis pubis with combined information from a lateral pelvic radiograph according to the method of Tannast et al. [11]. The crossover sign and the ISS then were assessed (Fig. 3). The intraobserver and interobserver observer kappa values for the crossover sign in patients have been reported $[1,7,11]$. The intraobserver kappa value ranged from 0.46 [1] to 0.83 [7]. The interobserver kappa value ranged from 0.39 [1] to 0.79 [11]. Based on these reports, only one observer (DKK) analyzed these radiographs.

To evaluate the diagnostic performance in the cadaver hips, a receiver operating characteristic curve (ROC) was calculated for tilt and rotation. The ROC curve is a graphic analytical technique that is used to evaluate the diagnostic performance of a test. Sensitivity is plotted on the y axis, and the false-positive rate $(1-$ specificity) is plotted on the $\mathrm{x}$ axis. The area under the ROC curve is a summary measure of the diagnostic performance of the test. A perfect test would approximate the upper left corner of the graph with an area under the curve of 1.0. Random guessing would be a straight line graph with an area under the curve of 0.5 . The area under the curve was interpreted 


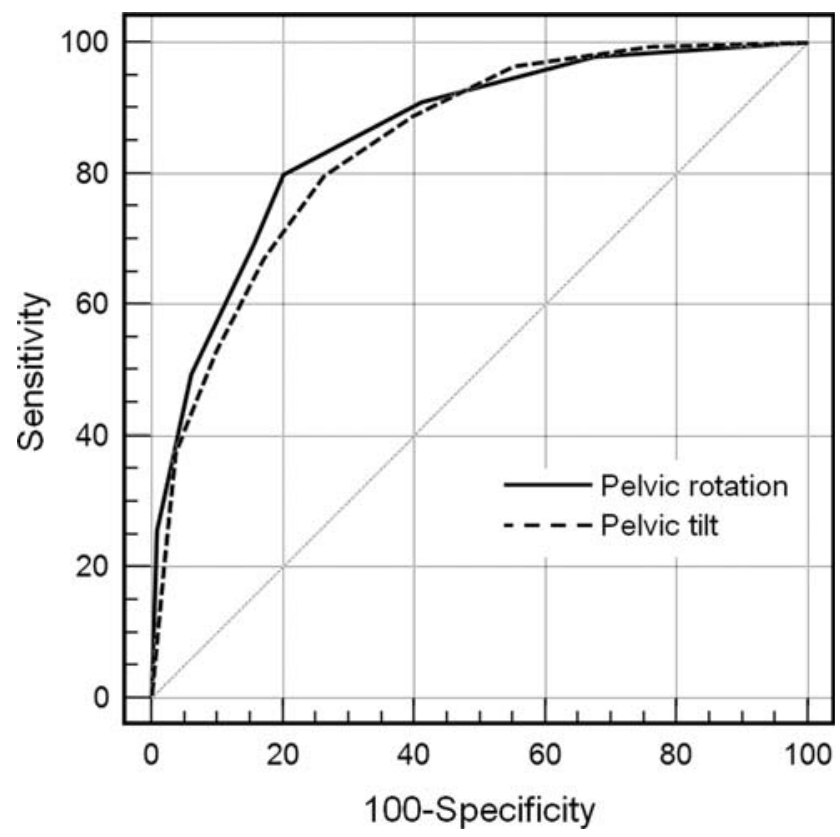

Fig. 3 The receiver operating characteristic curve (ROC) for the ISS as an indicator for apparent acetabular retroversion for the cadaver results is shown. The area under the ROC curve was judged as good for pelvic tilt and rotation.

as follows: 0.9 to 1 excellent, 0.9 to 0.8 good, 0.8 to 0.7 fair, and 0.7 to 0.6 poor. Contingency tables were created in which the ISS was listed against the apparent retroversion, which was considered to be the gold standard. Sensitivity, specificity, and positive and negative predictive values along with their $95 \%$ confidence intervals were calculated based on the presence or absence of the ISS as an indicator of the crossover sign for the cadaver and the patient series.

\section{Results}

For the cadaver radiographs, the area under the ROC curve was 0.845 for pelvic tilt and 0.860 for pelvic rotation indicating a good diagnostic performance of the ISS (Fig. 3). Sensitivity of ISS as a tool for detecting apparent retroversion was the measure with highest value for all
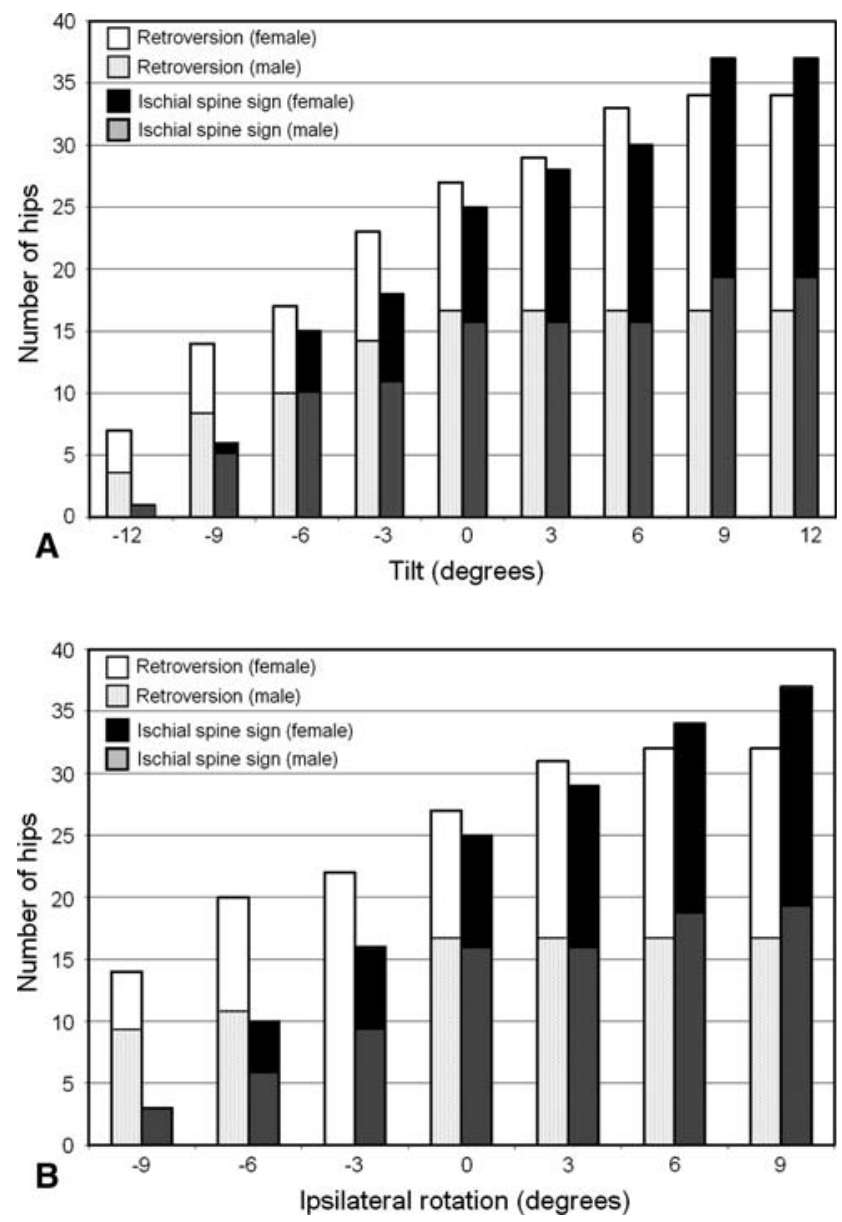

Fig. 4A-B (A) The presence of the ISS and acetabular retroversion with serially different pelvic tilts $(\mathrm{n}=40$ cadaver hips) and (B) with serially different pelvic rotations $(\mathrm{n}=40$ cadaver hips) are shown.

radiographs in general, for the male and female pelvic measurements, and for both situations of rotations involving interacetabular or longitudinal axis (Table 2). With increasing pelvic tilt and ipsilateral pelvic rotation, there was a corresponding increase in the ISS (Fig. 4). With a pelvic tilt of $-12^{\circ}$, only one of the 40 hips (3\%) showed a present ISS (Fig. 4A). In contrast, with a tilt of $+12^{\circ}, 37$ of the hips $(93 \%)$ showed a protrusion of the ischial spine and therefore a present ISS. On similar grounds, with a $-9^{\circ}$ ipsilateral pelvic rotation, three hips $(8 \%)$ showed a present

Table 2. Summary of the validity of the ISS for detection of acetabular retroversion in cadaveric specimens

\begin{tabular}{lllrr}
\hline Selection & Sensitivity $(\%)$ & Specificity $(\%)$ & PPV $(\%)$ & NPV $(\%)$ \\
\hline All radiographs $(\mathrm{n}=760 \mathrm{hips})$ & $81.3(95 \%$ CI, 0.77-0.84) & $69.5(0.64-0.74)$ & $76.5(0.72-0.80)$ & $75.3(0.70-0.79)$ \\
Male specimens $(\mathrm{n}=380 \mathrm{hips})$ & $78.5(0.72-0.83)$ & $63.2(0.54-0.70)$ & $77.2(0.71-0.82)$ & $65(0.56-0.72)$ \\
Female specimens $(\mathrm{n}=380)$ & $84.8(0.78-0.89)$ & $74.3(0.67-0.80)$ & $75.8(0.69-0.81)$ & $83.8(0.77-0.88)$ \\
Only tilted radiographs $(\mathrm{n}=320)$ & $85.2(0.79-0.89)$ & $69.3(0.61-0.76)$ & $77(0.70-0.82)$ & $79.5(0.71-0.85)$ \\
Only rotated radiographs $(\mathrm{n}=240)$ & $80.3(0.73-0.86)$ & $68.8(0.59-0.76)$ & $76.9(0.69-0.83)$ & $73(0.63-0.80)$ \\
\hline
\end{tabular}

$\mathrm{PPV}=$ positive predictive value; $\mathrm{NPV}=$ negative predictive value; $\mathrm{CI}=$ confidence interval. 
Table 3. Summary of the validity of the ISS for detection of acetabular retroversion in patients

\begin{tabular}{lllrr}
\hline Selection & Sensitivity $(\%)$ & Specificity $(\%)$ & PPV $(\%)$ & NPV $(\%)$ \\
\hline All radiographs $(\mathrm{n}=129)$ & $90.5(95 \%$ CI, 0.80-0.95) & $70.9(0.57-0.81)$ & $80.7(0.70-0.88)$ & $84.7(0.70-0.93)$ \\
Men $(\mathrm{n}=77)$ & $90.7(0.77-0.96)$ & $61.8(0.44-0.77)$ & $75(0.60-0.85)$ & $84(0.63-0.94)$ \\
Women $(\mathrm{n}=52)$ & $90.3(0.73-0.97)$ & $85.7(0.62-0.96)$ & $90.3(0.73-0.97)$ & $85.7(0.62-0.96)$ \\
\hline
\end{tabular}

$\mathrm{PPV}=$ positive predictive value; $\mathrm{NPV}=$ negative predictive value; $\mathrm{CI}=$ confidence interval.

Fig. 5A-D (A) A 38-year-old man with $-2^{\circ}$ pelvic tilt and neutral rotation had bilateral ISS and crossover sign. (B) A 24year-old man with $-4^{\circ}$ pelvic tilt and neutral rotation had bilateral negative ISS and crossover sign. The visible joint line (single line arrow) is the first joint of the coccyx. The sacrococcygeal joint (double line arrow) lies between the sacrum and the first vertebra of the coccyx. (C) A 34-year-old man with excessive pelvic tilt $\left(11^{\circ}\right)$ and neutral rotation had bilateral ISS and crossover sign. (D) A 29-year-old woman with neutral tilt and $5^{\circ}$ rotation to the left had ISS and crossover sign on the left, whereas negative values were found on the right.
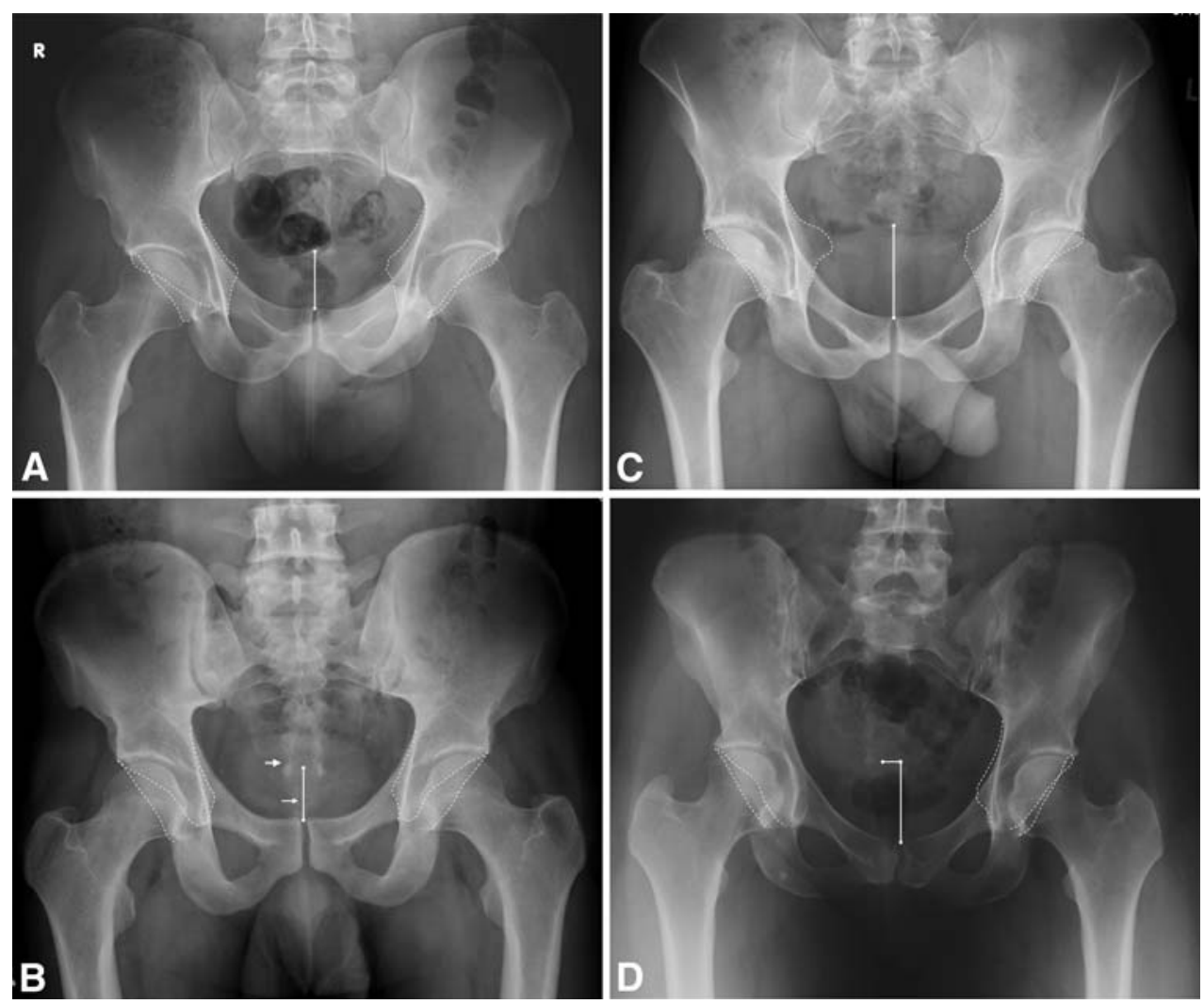

ISS, whereas with $+9^{\circ}$ pelvic rotation, 37 hips (93\%) had a present ISS (Fig. 4B).

In the review of clinical radiographs, sensitivity of the ISS as a measure was again the highest for all patient radiographs and for male and female genders (Table 3; Fig. 5).

\section{Discussion}

Retroversion of the acetabulum that is described as an abnormal morphologic feature involving a posteriorly oriented cranial acetabular opening with reference to the sagittal plane has been implicated as a cause for femoroacetabular impingement and subsequent osteoarthritis $[5,9]$. It has been suggested that a retroverted acetabulum is secondary to external or outward rotation of the entire distal hemipelvis [6-8]. Consequently, this externally rotated hemipelvis would lead to protrusion of the ischial spine into the true pelvis. Although a correlation between ISS and acetabular retroversion on plain radiographs is well known, it is not known whether the sign is reliable on pelvic radiographs with suboptimal pelvic orientation. Our aim therefore was to determine if the ischial spine sign is a valid sign for detecting apparent acetabular retroversion in pelves with variations of tilt and rotation.

In addition to the relatively low number of cadaver specimens, there are several limitations for our study. First, our results are only valid when the standardized radiographic technique is used that represents the current standard in imaging of femoroacetabular impingement. In particular, proper centering of the radiographic beam plays an important role in the projection of the ischial spine and both acetabular rims. A similar phenomenon is well described for projection of the cup orientation in THA [4]. Our results probably should not be extrapolated for 
radiographs in which the beam is centered over the hip and an acetabular retroversion potentially can be missed. Second, our results represent only radiographic measurements and interpretations without clinical information. However, the results of this study are crucial for further evaluation in larger clinical studies of the relevance of the ISS with various pelvic orientations to define the role of pelvic lordosis and the ability of the spine to accommodate for the pelvic position and the acetabular orientation dynamically. Third, our radiographs were not blinded. However, with the acetabular rim being wire-marked and the resulting high intraobserver and interobserver values, this should not compromise the conclusions of our study.

Our data differ in some ways from those of Kalberer et al. [7]. They noted a specificity of $91 \%$ and a sensitivity of $98 \%$ for the ISS as a diagnostic tool for acetabular retroversion. However, they excluded suboptimal oriented pelves. We found a similar specificity (92\%) to theirs when our cadaver pelvis specimens were in the neutral orientation. However, while considering all the possible malorientations that could occur, the specificity reduces to $71 \%$ and the sensitivity to $91 \%$ (Table 3). Nevertheless, the high sensitivity in the cadaver and the patient series indicates if a ISS is present, there is a high probability of a retroverted acetabulum. The corollary is not true. A negative ISS does necessarily exclude a crossover sign (the positive predictive value is only $71 \%$ ). We did not identify any consistent correlation between specific pelvic malorientation and a false-positive result. We believe the lower specificity most probably is the result of the individual morphologic features of the pelvis rather than pelvic malorientation.

We did not try to quantify the size of the ischial spine for several reasons. There is no scientific evidence to date of a direct relation between the amount of acetabular retroversion and symptoms or disease progression. Spearman's correlation coefficient between the size of the ischial spine and the retroversion was only 0.6 in the original study [7] when the measurements were not adjusted for magnification. Depending on the size of the patient, the same size of the ischial spine can lead to different appearances of the retroversion.

The presence of an ISS depends on the individual pelvic orientation as does acetabular retroversion. The more the tilt and ipsilateral rotation of the pelvis, the more the ischial spine will protrude into the true pelvis. The ISS is a valid sign for diagnosing acetabular retroversion independent of the pelvic tilt and rotation. The ISS could be used as a reliable sign to diagnose retroversion in various clinical situations, including poor-quality films, situations in which the anterior and posterior rims are not clearly visible, and in cases in which there is an element of pelvic tilt or rotation provided the standardized radiographic technique is used.

\section{References}

1. Clohisy JC, Carlisle JC, Trousdale R, Kim YJ, Beaule PE, Morgan P, Steger-May K, Schoenecker PL, Millis M. Radiographic evaluation of the hip has limited reliability. Clin Orthop Relat Res. 2009;467:666-675.

2. Drenckhahn D, Eckstein F. Lower extremity. In: Drenckhahn D, ed. Benninghoff Anatomy [in German]. München, Germany: Urban \& Fischer; 2003:342-350.

3. Ganz R, Parvizi J, Beck M, Leunig M, Nötzli H, Siebenrock KA. Femoroacetabular impingement: a cause for osteoarthritis of the hip. Clin Orthop Relat Res. 2003;417:112-120.

4. Georgen TG, Resnik D. Evaluation of acetabular anteversion following total hip arthroplasty: necessity of proper centring. $\mathrm{Br} J$ Radiol. 1975;48:259-260.

5. Giori NJ, Trousdale RT. Acetabular retroversion is associated with osteoarthritis of the hip. Clin Orthop Relat Res. 2003;417:263-269.

6. Jamali AA, Mladenov K, Meyer DC, Martinez A, Beck M, Ganz R, Leunig M. Anteroposterior pelvic radiographs to assess acetabular retroversion: high validity of the 'cross-over' sign. J Orthop Res. 2007;25:758-765.

7. Kalberer F, Sierra RJ, Madan SS, Ganz R, Leunig M. Ischial spine projection into the pelvis: a new sign for acetabular retroversion. Clin Orthop Relat Res. 2008;466:677-683.

8. Köhnlein W, Ganz R, Impellizeri FM, Leunig M. Acetabular morphology: implications for joint-preserving surgery. Clin Orthop Relat Res. 2009;467:682-691.

9. Reynolds D, Lucas J, Klaue K. Retroversion of the acetabulum: a cause of hip pain. J Bone Joint Surg Br. 1999;81:281-288.

10. Siebenrock KA, Kalbermatten DF, Ganz R. Effect of pelvic tilt on acetabular retroversion: a study on cadavers. Clin Orthop Relat Res. 2003;407:241-248.

11. Tannast M, Mistry S, Steppacher SD, Reichenbach S, Langlotz F, Siebenrock KA, Zheng G. Radiographic analysis of femoroacetabular impingement with Hip $^{2}$ Norm: reliable and validated. J Orthop Res. 2008;26:1199-1205.

12. Tannast M, Siebenrock KA, Anderson SE. Femoroacetabular impingement: radiographic diagnosis-what the radiologist should know. AJR Am J Roentgenol. 2007;188:1540-1552.

13. Tannast M, Zheng G, Anderegg C, Burckhard K, Langlotz F, Ganz R, Siebenrock KA. Tilt and rotation correction of acetabular version on pelvic radiographs. Clin Orthop Relat Res. 2005;438:182-190.

14. Williams PL. The skeleton of the lower limb. In: Williams PL, Warkick R, Dyson M, Bannister LH, eds. Gray's Anatomy. Edinburgh, Scotland: Churchill Livingstone; 1989:422-446. 